\title{
A State-Dependent Approximation for the Generalized Engset Model
}

\author{
Eric W. M. Wong, Senior Member, IEEE, Andrew Zalesky, and Moshe Zukerman, Fellow, IEEE
}

\begin{abstract}
The well-known Engset model has been widely used and studied. In this paper, we propose a new state dependent approximation for a special case of the generalized Engset model that considers packet/burst dumping. We also correct here several errors in [7] which considered the same model. Numerical results over a wide range of parameters demonstrate the superiority of the new approximation over previous ones.
\end{abstract}

Index Terms-Blocking probability, Engset formula, OBS, bufferless optical networks.

\section{INTRODUCTION}

$\mathbf{I}$ $\mathrm{N}$ this paper, we consider one of Cohen's generalizations of the Engset model [2], [4] in which the time before a source generates a new call is dependent on the success of the previous call. The concept of a call, which was relevant to telephony, was often used in classical work on the Engset model. In the context of modern networking research it represents packets or bursts. In this paper, we will use calls, packets or bursts, interchangeably. In our modeling, they all refer to jobs offered to a service system.

This generalization is applicable to optical burst (or packet) switching [1], [3], [8], in which a blocked burst is dumped, and until the dumping is completed, a source cannot generate a new burst. The salient feature that distinguishes such a special case from the traditional Engset model is that a source must completely transmit (i.e. dump) a blocked burst before a new burst can be generated. In contrast, the Engset model assumes a new burst can be generated immediately after a blocking event which results in blocking probability overestimation. Therefore, it was proposed in [5], [6], [7] to increase the source's off-time to capture the burst dumping effect.

In this paper, for a given source, the mean effective offtime (MEOT) refers to the mean time period between the end of transmission of a successful burst and the commencement of transmission of the next successful one. This period may incorporate the dumping of blocked bursts. We consider here MEOT for each source to be dependent on the number of busy channels, and demonstrate that this leads to an approximation which is consistently more accurate than all previous proposals. In addition, we correct several errors made in [7].

Manuscript received May 22, 2009. The associate editor coordinating the review of this letter and approving it for publication was M. Ma.

E. W. M. Wong and M. Zukerman are with the Department of Electronic Engineering, City University of Hong Kong, Hong Kong SAR, China (e-mail: ewong@ee.cityu.edu.hk; m.zu@cityu.edu.hk).

A. Zalesky is with the University of Melbourne, Victoria 3010, Australia (e-mail: azalesky@unimelb.edu.au).

The work described in this paper was supported by a grant from City University of Hong Kong (Project No. 7002478).

Digital Object Identifier 10.1109/LCOMM.2009.091115

\section{THE MODEL}

We consider the loss model of [7], [8] involving $N$ sources that offer calls/packets/bursts to $K$ channels/servers. Both $N$ and $K$ are assumed to be finite integers. We assume the case $N>K$, but we do not consider the case $N>>$, which degenerates to the Erlang system as $N$ approaches infinity. On the other hand, if $N \leq K$, the call-congestion is equal to zero. Neither the case $N>>K$, nor the case $N \leq K$, is of interest to us here. We assume that the packet transmission or dumping times are independent and exponentially distributed with mean $1 / \mu$. At any point in time, any of the $N$ sources is either active, idle or dumping. During an active period, a source will transmit a successful packet. A source cannot generate a new packet during either an active or a dumping period. The end of an active or dumping period is always a beginning of an idle period. The length of an idle period is assumed to be independent and exponentially distributed with mean $1 / \lambda$. As soon as an idle period ends, the source generates a new packet. The new packet is blocked if all $K$ channels are already busy, which results in a dumping period. Otherwise, the new packet is successful, which results in an active period for that source.

\section{A StATE-DEPENDENT APPROXIMATION}

The aim here is to find the MEOT for each source which is dependent on the number of busy servers such that a single dimensional Markov chain can be used to accurately predict the blocking probability. Having an accurate approximation based on a single dimension is important because exact results for the generalized Engset relies on a 2-dimensional Markov chain that are not scalable for large $k$ and $N$. Note that although significant computational improvement can be achieved using Matrix methods [1], they are still not as scalable as a single dimensional solution.

All the approximations considered in [7] were based on lengthening the off-period from $1 / \lambda$ to $m$ to compensate for the time that packets or bursts are dumped immediately following a blocking event. However, they all do not consider the fact that during periods of higher carried traffic load there are more blocking and thus more dumping, so the MEOTs are longer. Therefore, we propose here to achieve higher accuracy by considering the MEOT to be state-dependent. To this end, we define $m_{i}$ to be the MEOT in state $i$, namely when there are $i$ busy servers, $i=0,1,2, \ldots, K$.

Note that in the traditional Engset model, bursts arrive at rate $(N-i) / m_{i}$ in state $i$, where $m_{i}=m=1 / \lambda$ for all $i$. The traditional model is therefore typically considered to be state-dependent but the MEOT (for each source) is stateindependent. In our new model, we allow the MEOT to be dependent on system state $i$ (i.e. $m_{i} \neq m$ ). 
Let $\pi_{i}$ be the probability of having $i$ servers busy, $i=$ $0,1,2, \ldots, K$. The $\pi_{i}$ and $m_{i}$ values satisfy the following steady state equations.

$$
\pi_{i+1}=\frac{N-i}{\mu m_{i}(i+1)} \pi_{i}, \text { for } i=0,1,2, \ldots, K-1,
$$

where the $m_{i}$ values are set to increase linearly with $i$ as follows

$$
m_{i}=\frac{1}{\lambda}+\frac{(i+1) a}{K \mu} .
$$

The choice of a linear relationship between $m_{i}$ and the state $i$ is made for simplicity. The constant $a$ is obtained as follows.

$$
\frac{\lambda}{\lambda+\mu} N=\sum_{i=0}^{K} \frac{(N-i)}{m_{i} \mu} \pi_{i} .
$$

In the real system, an idle period of mean length $1 / \lambda$ is always followed by a transmission/dumping period of mean length $1 / \mu$. The true burst arrival rate per source is therefore $1 /(1 / \mu+1 / \lambda)$ and is thus independent of the blocking probability. The left hand-side of (3), which is given by multiplying this expression by $N / \mu$, is the intended offered load in the real system.

In (3), we equate the intended offered load, namely the offered load in the real system of the on-off sources (including the dumped packets/bursts) to the offered load in the model.

Substituting (2) into (1) and (3) and together with the normalization equation $\sum_{i=0}^{K} \pi_{i}=1$, we have $K+2$ equations and $K+2$ unknowns (i.e. $a$ and $\pi_{i}$ where $i=0,1, \ldots, K$ ) which form a set of fixed point equations and can be solved by the algorithm provided in the next section.

The blocking probability is then derived by

$$
\Pi_{B}=1-\frac{\text { carried load }}{\text { offered load }}
$$

where carried load $=\sum_{i=0}^{K-1} \frac{(N-i)}{m_{i} \mu} \pi_{i}$.

In the remaining part of this section, we prove that a unique solution exists for the set of coupled fixed-point equations defined by (1), (2) and (3) together with the normalization equation. This solution is our new state-dependent approximation for call congestion. As we cannot guarantee that the successive substitution algorithm [5] converges to the unique solution, we provide a binary search algorithm to numerically compute the unique solution for the set of fixedpoint equations and we prove its convergence. Finally, in the next section, we numerically compare the accuracy of our new state dependent approximation with Syskis approximation and the approximations in [5] and [7].

\section{A. Existence and Uniqueness of the Fixed Point Solution}

By setting $x=a$ in (2) and moving the left-hand side of (3) to the right, we define the function

$$
f(x)=\sum_{i=0}^{K} \frac{(N-i)}{m_{i}(x) \mu} \pi_{i}-\frac{\lambda}{\lambda+\mu} N,
$$

where we have written $m_{i}(x)$ instead of $m_{i}$ to emphasize that $m_{i}$ is functionally dependent on $x$ through (2). Our task is to prove $f(x)=0, x \geq 0$, has a unique solution.

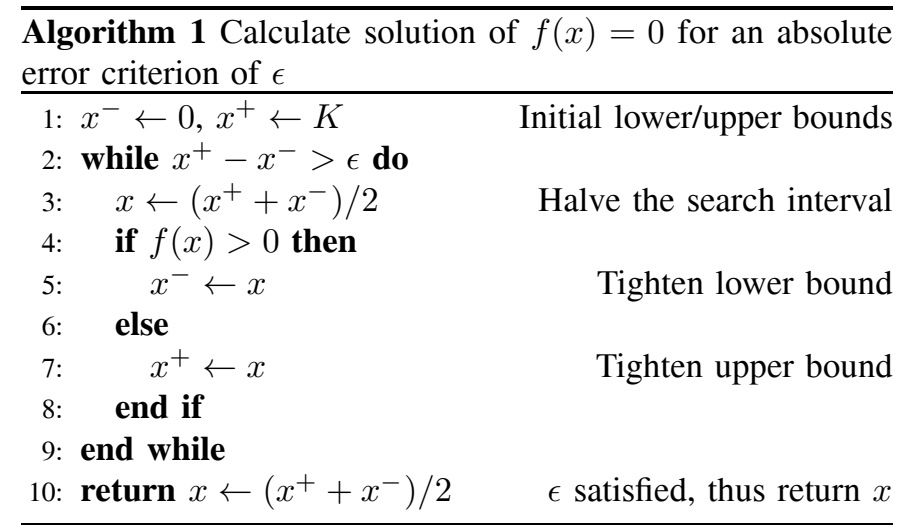

To establish solution existence, we observe that $f(x)$ is continuous and changes sign at least once for $x \geq 0$. This is because for $x=0, m_{i}=1 / \lambda$ and the system degenerates to the Engset system and hence $f(0)>0$ since in the Engset system the offered load is larger than the intended offered load for $\Pi_{B}>0$. On the other hand, for $x=K$

$$
\begin{aligned}
f(K) & =\sum_{i=0}^{K} \frac{(N-i)}{\left(\frac{1}{\lambda}+\frac{i+1}{\mu}\right) \mu} \pi_{i}-\frac{\lambda}{\lambda+\mu} N, \\
& <\sum_{i=0}^{K} \frac{N}{\left(\frac{1}{\lambda}+\frac{1}{\mu}\right) \mu} \pi_{i}-\frac{\lambda}{\lambda+\mu} N=0
\end{aligned}
$$

Therefore, a solution exists by the intermediate-value theorem.

To establish solution uniqueness, suppose $f\left(x_{1}\right)=f\left(x_{2}\right)=$ 0 for $x_{2}>x_{1} \geq 0$. The mean-value theorem requires the existence of an $\eta$ satisfying $f\left(x_{2}\right)-f\left(x_{1}\right)=f^{\prime}(\eta)\left(x_{2}-x_{1}\right)$ or $f^{\prime}(\eta)=0$, where $x_{1} \leq \eta \leq x_{2}$. This provides us with a contradiction because simple calculations reveal $f^{\prime}(x)<0$ (the proof is provided in the Appendix). Therefore, $x_{1}=x_{2}$.

\section{B. Binary Search Algorithm to Solve $f(x)=0$}

This is the well known binary search algorithm. The only reason we present it here is to correct our errors in the same algorithm introduced in [7]. Let $x^{*}$ be the unique solution of $f(x)=0$. Due to the monotonicity of $f(x)$, at each iteration of Algorithm 1, if $x<x^{*}$, then $f(x)>f\left(x^{*}\right)=0$. Conversely, if $x>x^{*}$, then $f(x)<f\left(x^{*}\right)=0$. Consequently, $x^{*}$ lies in the interval $\left[x^{-}, x^{+}\right]$at each iteration of Algorithm 1. Furthermore, this interval halves at each iteration, thereby ensuring $x^{*}$ is sandwiched within an interval whose eventual length does not exceed $\epsilon$. Thus, Algorithm 1 converges to a unique solution of $f(x)=0$ with error $\epsilon$.

\section{Numerical Results}

Figs. 1 and 2 show blocking probability versus normalized traffic intensity $\rho=(N / K)(\lambda / \mu)$ for $N=2$ and $K=1$ and for $N=6$ and $K=3$, respectively. These figures demonstrate that the new state-dependent (SD) approximation is consistently more accurate than all previous proposals. See [9] for further numerical results that demonstrate advantages of the MEOT state-dependent approach. 


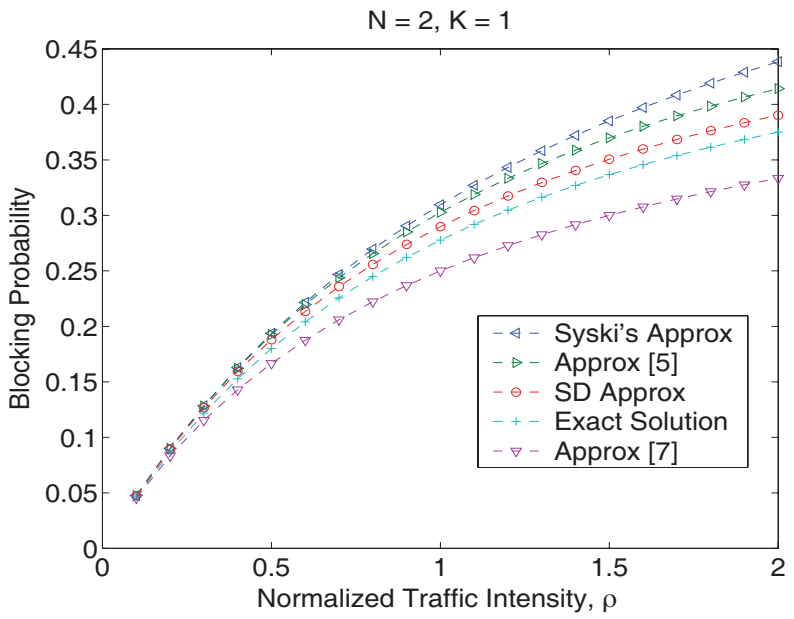

Fig. 1. Blocking probability versus normalized traffic intensity $\rho$ for $N=2$ and $K=1$.

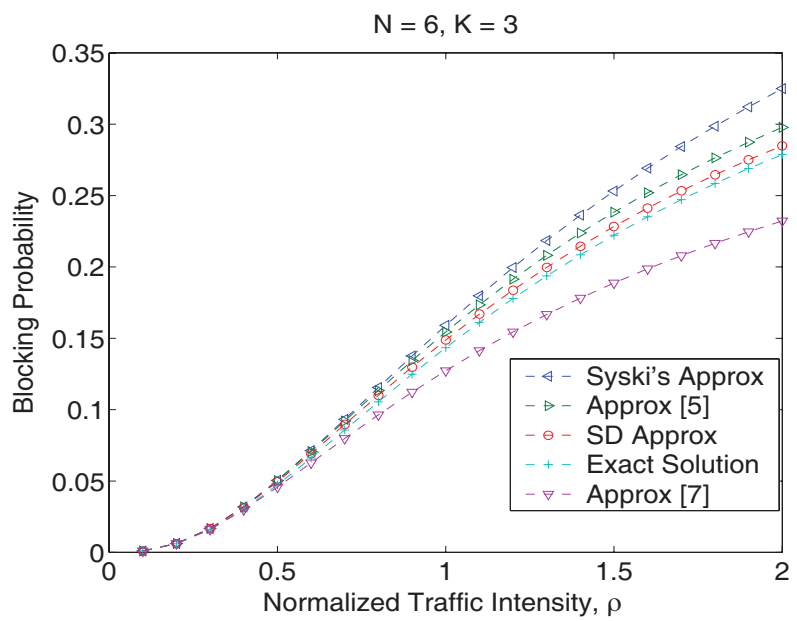

Fig. 2. Blocking probability versus normalized traffic intensity $\rho$ for $N=6$ and $K=3$.

\section{Corrections to [7]}

In lines 5 and 7 in Algorithm 1 in the 3rd page of [7], the updated $x^{-} / x^{+}$should be set to $x$ instead of $\Gamma(x)$. This change ensures the algorithm is a true "Binary Search Algorithm" as claimed in [7]. It guarantees convergence in a bounded number of steps. We cannot prove (or disprove) convergence if this change is not made. Also, some of the values reported in reported in Table 1 of [7] are in error. Specifically, all the rows in Table 1 listed as $\rho=0.9$ actually correspond to $\rho=0.8$. And the entire "New" column is in error due to a programming bug. This resulted in incorrect conclusions reported in [7] about the superiority of the "New" approximation.

\section{CONCLUSION}

We have provided a new MEOT state-dependent approximation and corrected several errors in [7] for a version of the generalized Engset model that considers packet/burst dumping. We have demonstrated numerically that the new approximation is consistently more accurate than previous proposals.

ApPendix: Proof of $f^{\prime}(x)<0$

Recall $m_{i}=m_{i}(x)=\frac{1}{\lambda}+\frac{i+1}{K} \frac{x}{\mu} \cdot f(x)$ can be written as

$$
\begin{aligned}
f(x) & =\sum_{i=0}^{K} \frac{(N-i)}{m_{i} \mu} \pi_{i}-\frac{\lambda}{\lambda+\mu} N \\
& =\frac{(N-K)}{m_{K} \mu} \pi_{K}+\sum_{i=1}^{K} i \pi_{i}-\frac{\lambda}{\lambda+\mu} N \\
& =\frac{(N-K)}{m_{K} \mu} \pi_{K}+\sum_{i=1}^{K}\left(\sum_{k=i}^{K} \pi_{k}\right)-\frac{\lambda}{\lambda+\mu} N .
\end{aligned}
$$

Proving $f(x)$ is decreasing in $x$ or $a$ means proving $\sum_{k=i}^{K} \pi_{k}$ is decreasing in $m_{k}$ since $m_{k}$ is increasing in $x$. Since $\pi_{j}=$ $\left(\prod_{k=j}^{K} \alpha_{k} m_{k}\right) \pi_{K}$ where $j=0,1,2, \ldots, K$ and $\alpha_{k} \triangleq$ $\frac{(k+1) \mu}{N-k}$ for $k=0,1,2, \ldots, K-1$ or $\triangleq 1 / m_{K}$ for $k=K$, we have

$$
\begin{aligned}
\sum_{k=i}^{K} \pi_{k} & =\frac{\sum_{j=i}^{K}\left(\prod_{k=j}^{K} \alpha_{k} m_{k}\right)}{\sum_{j=0}^{K}\left(\prod_{k=j}^{K} \alpha_{k} m_{k}\right)}=\frac{1}{\frac{1}{\sum_{j=0}^{i-1}\left(\prod_{k=j}^{K} \alpha_{k} m_{k}\right)}+1} \\
& =\frac{1}{\frac{\sum_{j=0}^{i-1}\left(\prod_{k=j}^{K} \alpha_{k} \alpha_{k}\right) /\left(\prod_{k=i}^{K} m_{k}\right)}{\sum_{j=i}^{K}\left(\prod_{k=j}^{K} \alpha_{k} m_{k}\right) /\left(\prod_{k=i}^{K} m_{k}\right)}+1} \\
& =\frac{1}{\frac{\sum_{j=0}^{i-1}\left(\prod_{k=j}^{K} \alpha_{k}\right)\left(\prod_{k=j}^{i-1} m_{k}\right)}{\sum_{j=i}^{K}\left(\prod_{k=j}^{K} \alpha_{k}\right)\left(\prod_{k=i}^{j-1} \frac{1}{m_{k}}\right)}+1}
\end{aligned}
$$

where $i=1,2, \ldots, K$ and $\prod_{k=i}^{i-1} \frac{1}{m_{k}} \triangleq 1$. Since $\alpha_{k}>0, \sum_{j=0}^{i-1}\left(\prod_{k=j}^{K} \alpha_{k}\right)\left(\prod_{k=j}^{i-1} m_{k}\right)^{m_{k}}$ is increasing in $m_{k}$ and $\sum_{j=i}^{K}\left(\prod_{k=j}^{K} \alpha_{k}\right)\left(\prod_{k=i}^{j-1} \frac{1}{m_{k}}\right)$ is decreasing in $m_{k}$, and hence $\sum_{k=i}^{K} \pi_{k}$ is decreasing in $m_{k}$. Therefore, $f(x)$ is decreasing in $x$ or $a$.

\section{REFERENCES}

[1] N. Akar and Y. Gunalay, "Stochastic analysis of finite population bufferless multiplexing in optical packet/burst switching systems," IEICE Trans. Commun., vol. E90-B, no. 2, pp. 342-345, 2007.

[2] J. W. Cohen, "The generalized Engset formulae," Philips Telecommun. Rev., vol. 18, pp. 158-170, 1957.

[3] H. Overby, "Performance modelling of optical packet switched networks with the Engset traffic model," Optics Express, vol. 13, pp. 1685-1695, 2005.

[4] R. Syski, Introduction to Congestion Theory in Telephone Systems. North Holland, 1959.

[5] H. L. Vu et al., "Scalable performance evaluation of a hybrid optical switch," J. Lightwave Technol., vol. 23, pp. 2961-2973, Oct. 2005.

[6] E. W. M. Wong and M. Zukerman, "Bandwidth and buffer tradeoffs in optical packet switching," J. Lightwave Technol., vol. 24, no. 12, pp. 4790-4798, Dec. 2006.

[7] E. W. M. Wong, A. Zalesky, and M. Zukerman, "On generalizations of the Engset model," IEEE Commun. Lett., vol. 11, no. 4, pp. 360-362, Apr. 2007.

[8] M. Zukerman, E. W. M. Wong, Z. Rosberg, G. M. Lee, and H. L. Vu, "On teletraffic application to OBS," IEEE Commun. Lett., vol. 8, pp. 116-118, Feb. 2004.

[9] A. Zalesky, E. W. M. Wong, M. Zukerman, and H. L. Vu, "Engset formula for bufferless OBS/OCS: when is and when isn't lengthening the off-time redundant?" Proc. IEEE GLOBECOM 2009, to be published. 